\title{
Modeling of $\gamma$ field around irradiated TRIGA fuel elements by R2S method
}

\author{
Ambrožič Klemen ${ }^{1, *}$ and Snoj Luka ${ }^{1, * *}$ \\ ${ }^{1}$ Jožef Stefan Institute, Jamova cesta 39, SI-1000 Ljubljana, Slovenia
}

\begin{abstract}
The JSI TRIGA reactor has several irradiation facilities with well characterized neutron fields. The characterization was performed by measurements and by utilizing Monte Carlo particle transport computational methods. Because of this, JSI TRIGA has become a reference center for neutron irradiation of detectors for ATLAS experiment (CERN). Thorough $\gamma$ characterization of the reactor is however yet to be performed. Current Monte Carlo particle transport code only account for the prompt generation of neutron induced $\gamma$ rays, which have been characterized, but are neglecting the time dependent delayed part, which may in some cases amount to more then $30 \%$ of total $\gamma$ flux in an operation reactor, and is the only source of $\gamma$-rays after reactor shutdown. Several common approaches of modeling delayed $\gamma$-rays, namely D1S and R2S exist.

In this paper an in-house developed R2S method code is described, coupling a Monte Carlo particle transport code MCNP6 and neutron activation code FISPACT-II, with intermediate steps performed by custom Python scripts.

An example of its capabilities is presented in terms of evaluation of utilization of JSI TRIGA nuclear fuel as a viable $\gamma$-ray source. In the model, fresh nuclear fuel is considered and a silicon pipe sample is modeled in. Fuel activities, dose and kerma rates on the sample, as well as emitted $\gamma$-ray spectra and isotopic contribution to the contact dose are calculated and presented.
\end{abstract}

\section{Introduction}

The Jožef Stefan Institute (IJS) TRIGA reactor is a $250 \mathrm{~kW}$ pool type reactor featuring numerous irradiation facilities with different characteristics in terms of size and neutron field properties [1]. Neutron fluxes and spectra in these facilities have been well characterized both computationally by Monte Carlo computational models [1] and experimentally validated [2]. Due to this, JSI TRIGA has become a reference center for neutron irradiation of detectors for ATLAS experiment (CERN) [3] within the AIDA project [4].

However this is not the case for $\gamma$-ray fields. Event though particle transport computer codes, such as Monte Carlo code MCNP support generation of neutron induced $\gamma$-rays, commonly only prompt $\gamma$-ray generation is supported by default [5]. Measurement with ionization chamber indicate that $\gamma$ ray fluxes due to decay of neutron activated isotopes (delayed $\gamma$-rays) contribute up to $30 \%$ of total photon flux $[6,7]$. Additionally these $\gamma$-ray fluxes are time dependent due to decay of fission and

*e-mail: klemen.ambrozic@ijs.si

**e-mail: luka.snoj@ijs.si 
activation products and are also present after reactor shutdown. Up to date, only prompt $\gamma$ fields have been characterized [5], with delayed part still to be evaluated.

Two approaches in Monte Carlo particle transport for modeling delayed $\gamma$-rays, namely D1S [8] and $\mathrm{R} 2 \mathrm{~S}[9,10]$ are used. Initial steps on delayed $\gamma$ ray characterization have been performed utilizing an in-house developed R2S method code on TRIGA nuclear fuel, to assess feasibility of its use as a viable $\gamma$-source. The code is written in Python 2.7 (Python) [11] and couples MCNP [12] particle transport capabilities with FISPACT-II [13] neutron activation and transmutation analysis computer code.

In the calculations fresh nuclear fuel is considered, and two activation time regimes are evaluated: short irradiation in terms of several hours at full reactor power which simulates intra-day reactor operation with emphasis on observing short-lived isotopes, and long term irradiation with fuel element burn-up of $1 \mathrm{MWd}$ and $10 \mathrm{MWd}$, which is representative of several years of reactor operation and account for long-lived isotopes accomulation.

Calculations on JSI TRIGA fuel elements pose several advantages, due to the fact the operational history of every fuel element is known and easy acessibility of reactor core and fuel elements inside the reactor core, allowing for easy acess for experimental validation of the computational data, which will be performed in the next two years.

The current code is problem specific, but several of its modules could be used in a more generalized code, with possible cell under voxel approach [10], and as such offers significant insight in the method performance capabilities. A generalized version of the code is currently being developed and will be verified with an extensive experimental campaign.

\section{Rigorous two-step method (R2S)}

R2S [9] in reactor physics is a method of coupling particle transport capabilities with neutron activation calculations for characterization of delayed $\gamma$-ray emissions. The basic idea is, to divide the geometry of the computational model into small voxels, and calculate neutron flux and spectrum in each of them. This, along with initial material composition of the voxel is input into the activation analysis computer code, generating isotope inventory as well as $\gamma$-ray spectra respectively. The latter is then used as input into particle transport computer code again, so each voxel is represented in terms of a $\gamma$ source respectively, calculating delayed photon field flux, spectrum and dose. Such approach allows user to calculate for example dose fields around activated or irradiated samples or in our case around irradiated nuclear fuel. The approach is commonly used in assessing radiation fields in ITER [9].

Our Python script couples MCNP6 [12] particle transport code with neutron activation and transmutation analysis code FIPACT-II [13] in the above mentioned fashion. MCNP model geometry is divided into voxels and in each voxel neutron flux and spectra are calculated using cell averaged track length neutron flux estimator (F4 in MCNP) and normalization to desirable reactor power [1].

Material composition and time dependent neutron flux in terms of normalized neutron spectra and total neutron fluxes are input into the activation calculations. Results of activation calculations are time dependent isotopic compositions of activated samples and gamma rays arising from activated material. The latter are then used as input source in MCNP model, where $\gamma$-ray transport is performed allowing user to calculate gamma flux and dose fields. Dose fields were calculated in term of ICRP$21 H^{*} 10$ dose [14] using flux-to-dose conversion factors and kerma using energy deposition over cell estimates (F6 in MCNP) are calculated. Several intermediate data conversion tasks are performed by the Python script for data input/output (I/O) compatibility between computer codes. A flow chart of script operation is presented in figure 1. 


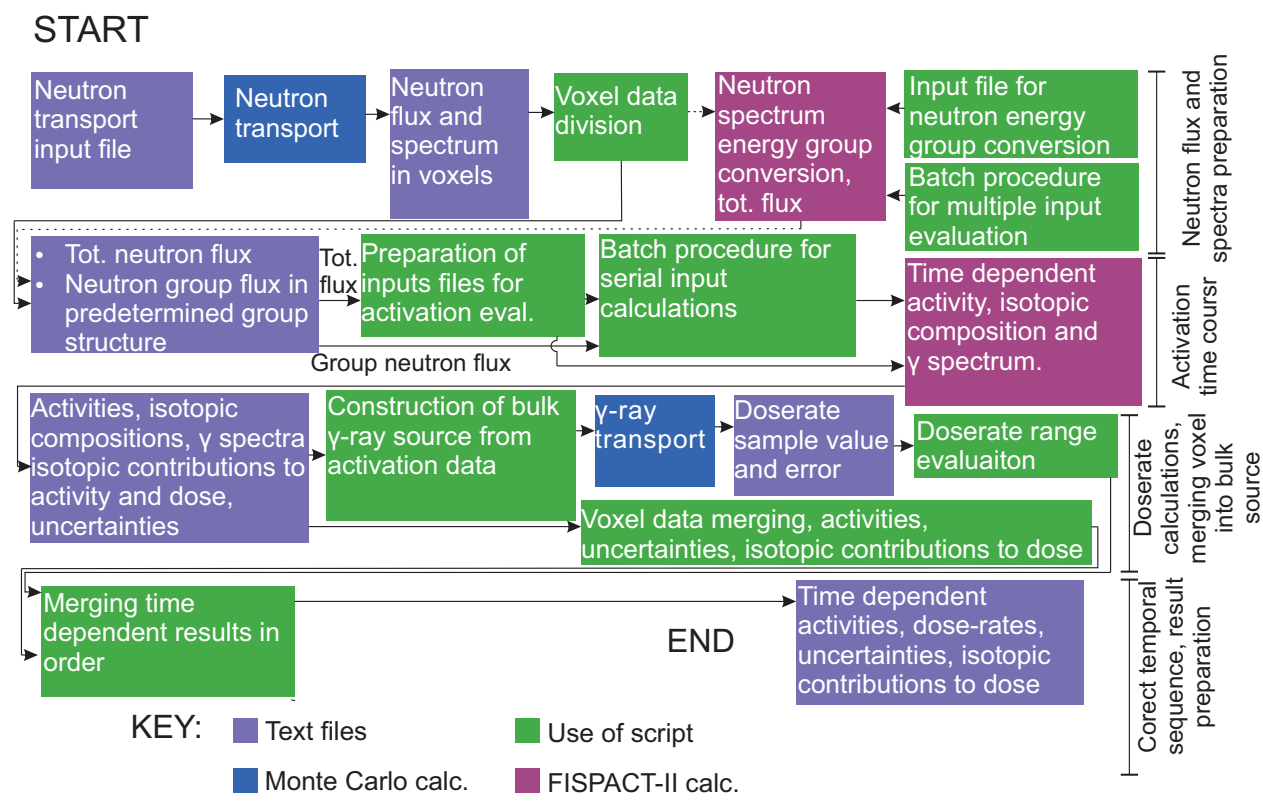

Figure 1: Flow chart of the R2S script operation.

\section{JSI TRIGA fuel element R2S evaluation of delayed $\gamma$-ray dose.}
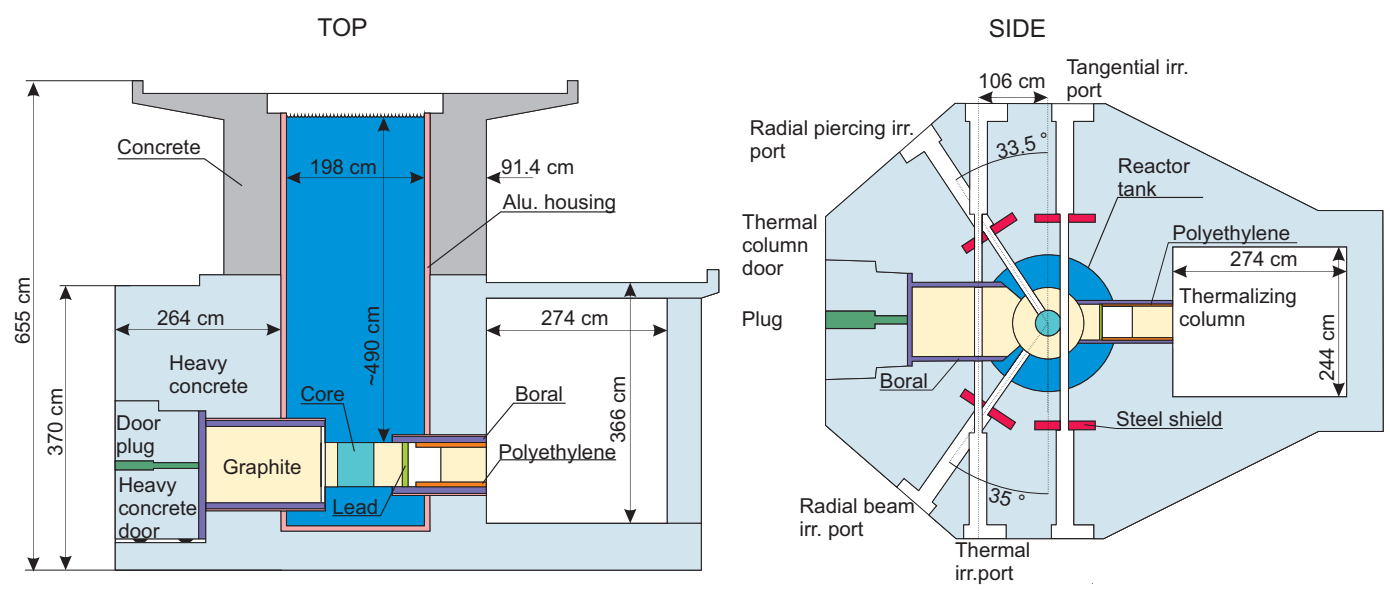

Figure 2: JSI TRIGA reactor, top and side schematic view.

Since activated nuclear fuel is highly radioactive, its utilization as a viable $\gamma$-ray source was calculated using R2S method. JSI TRIGA is a pool type, $250 \mathrm{~kW}$ reactor 2, with fuel elements arranged in an annular configuration in the reactor core 3 . Each fuel element comprises of $\mathrm{Zr}$ rod, with homogeneous mixture of uranium-zirconium hydride alloy $(\mathrm{U}-\mathrm{ZrH})$ fuel/moderator and stainless-steel cladding 4. Several representative, standard fuel elements containing $12 \mathrm{wt} \%$ of $\mathrm{U}$ in $\mathrm{U}-\mathrm{ZrH}$ (Uranium enrichment is $20 \%$ ) were selected for our calculations. $\mathrm{U}-\mathrm{ZrH}$ part of each of the fuel elements was divided 
into 250 small voxels ( 10 axially $\times 5$ radially $\times 5$ azimuthally) depicted on fig. 4 , and the above mentioned R2S calculation method performed on them. Time dependency of $\gamma$-ray spectra and intensities was evaluated in each voxel, and a bulk photon source constructed respectively. Since an increased

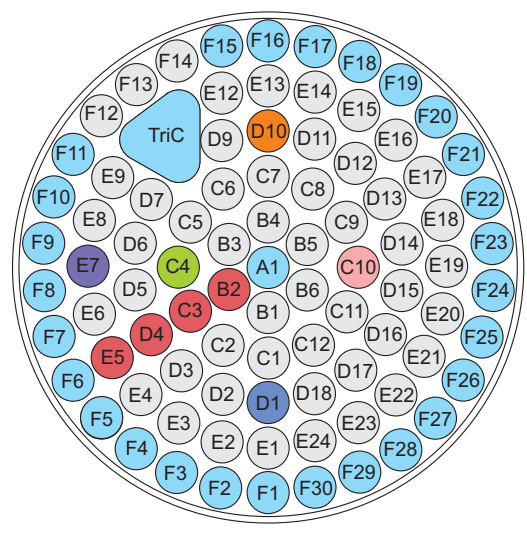

$20 \%$ LEU fuel element

Empty/irradiation in-core position

Representative fuel element

Neutron source

Compensating control rod

Shim control rod

Regulating control rod

Pulse control rod

Figure 3: JSI TRIGA core with typical fuel element and control rod arrangement and incore position annotation.

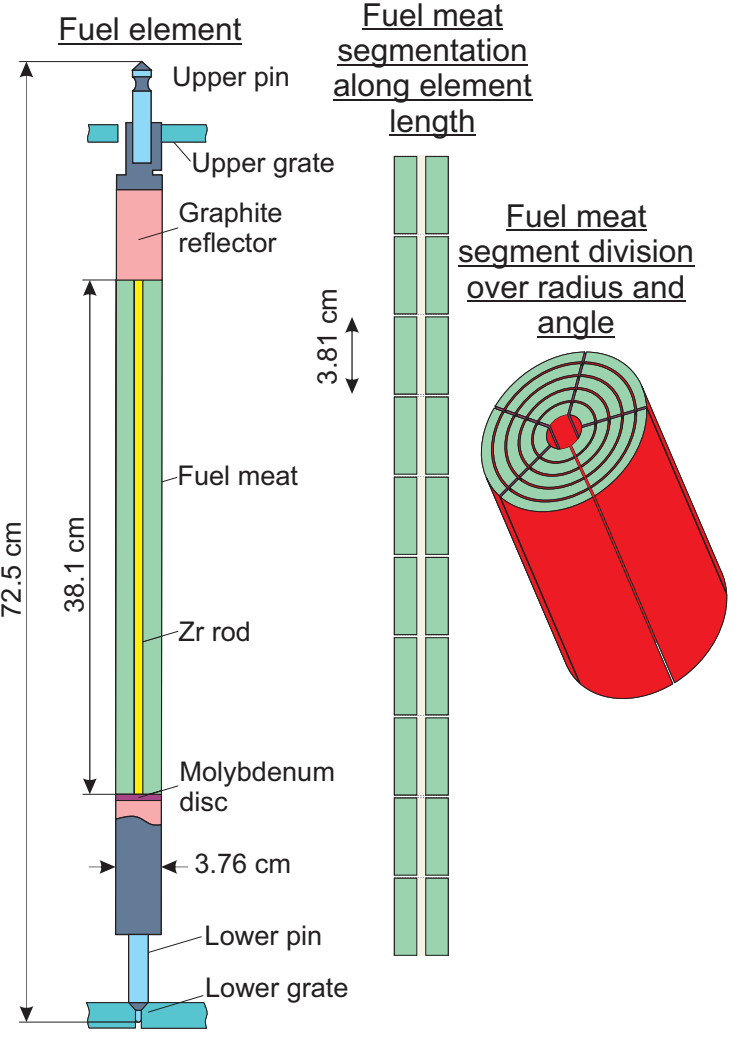

Figure 4: JSI TRIGA fuel element and fuel meat division in 250 voxels for $\mathrm{R} 2 \mathrm{~S}$ calculation.

interest in semiconductor testing against high dose and dose-rate of $\gamma$ radiation has been observed, a pipe silicon sample surrounding the fuel element has been modeled in this particular computational model (fig. 6). A 3D mesh for track length-based estimator of the photon fluence, kerma and $H^{*} 10$ dose using ICRP-21 [14] flux to dose conversion coefficients has been superimposed over the sample geometry. For the initial step, 100-group neutron spectra has been calculated in each of the fuel element meat voxels, to obtain the neutron activation software data (fig. 5).

Two distinct fresh TRIGA fuel irradiation time regimes were evaluated, for irradiation times of several hours and for $1 \mathrm{MWd}$ and $10 \mathrm{MWd}$ burn-ups. For irradiation times of several hours, operation at full reactor power for an appropriate amount of time is simulated. For longer irradiations, until a fuel element reaches burn-up of $1 \mathrm{MWd}$ or $10 \mathrm{MWd}, 40 \mathrm{~h} /$ week, $8 \mathrm{~h} \mathrm{~d}^{-1}$ operation at full power is simulated. The results are presented in terms of fuel element activities, $H^{*} 10$ and kerma on the sample, as well as fuel element delayed $\gamma$ spectra and isotopic contribution to contact dose vs. cooling time after activation completion respectively. 


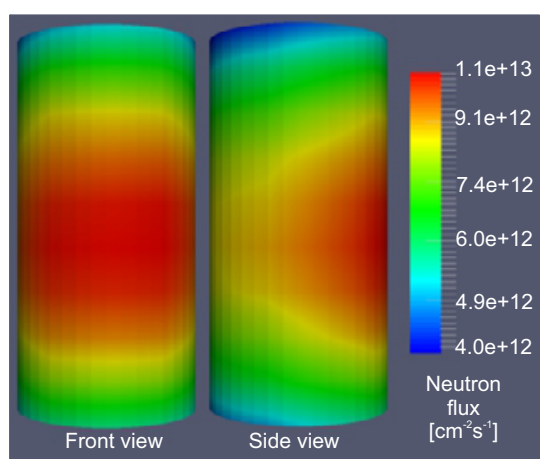

Figure 5: Total neutron flux distribution on the surface of the active part of the $\mathrm{B} 2$ position fuel element.

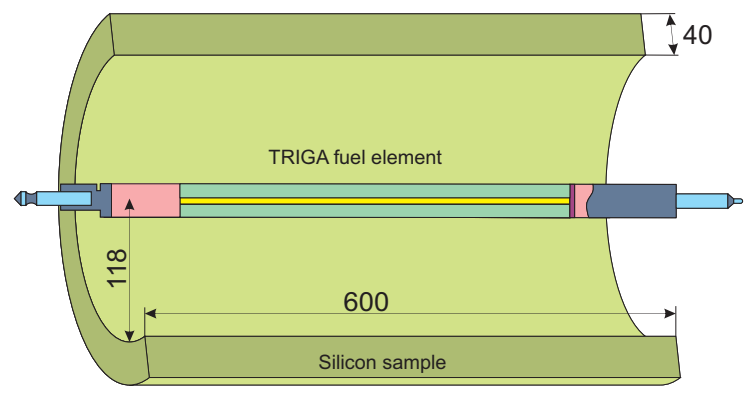

Figure 6: JSI TRIGA fuel element and the silicon pipe sample model schematic.

\subsection{Irradiation times of several hours}

The case of fuel element neutron irradiation for several hours corresponds to day to day reactor operations, which is the most convenient way of sufficiently activating the nuclear fuel, and use it as a viable $\gamma$ source. Fresh nuclear fuel is considered. Kerma rate and dose rate to the silicon sample are given in range terms, due to large gradients of photon fields inside the silicon sample itself. The computational uncertainty (not accounting for the nuclear data uncertainty) is in all cases below $1 \%$.

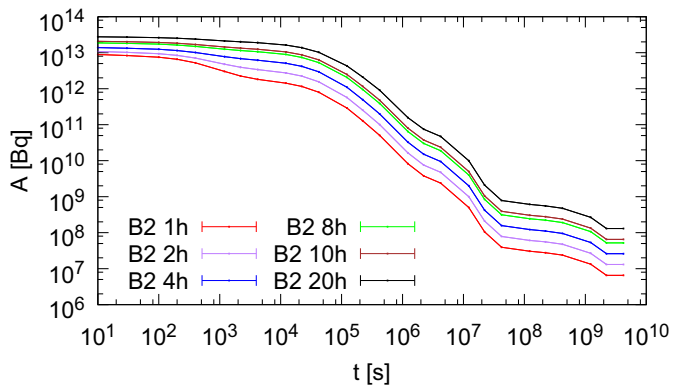

(a) B2 fuel element activity.

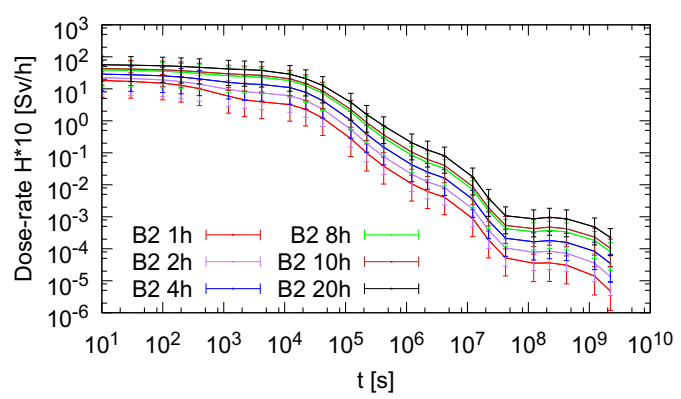

(c) B2 fuel element dose-rate to sample.

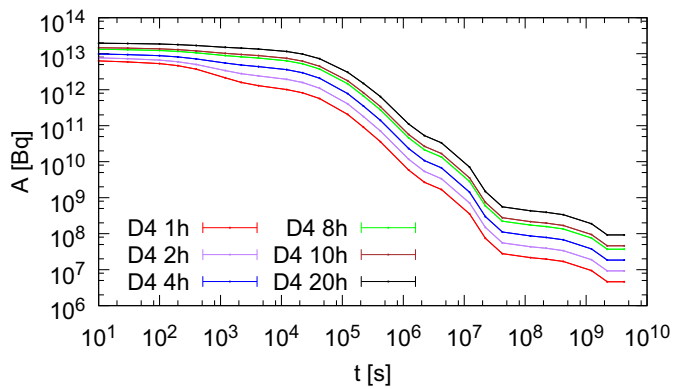

(b) D4 fuel element activity.

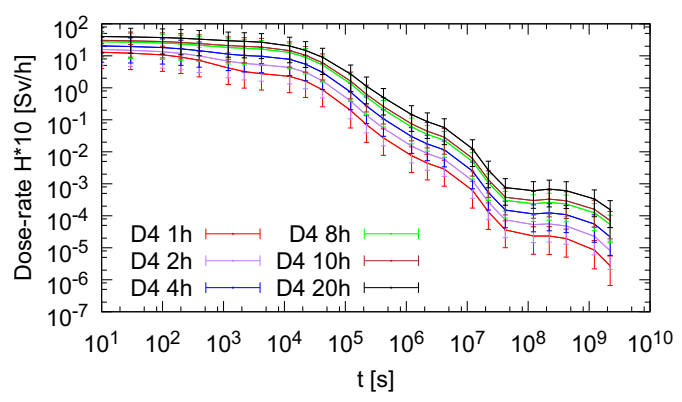

(d) D4 fuel element dose-rate to sample. 


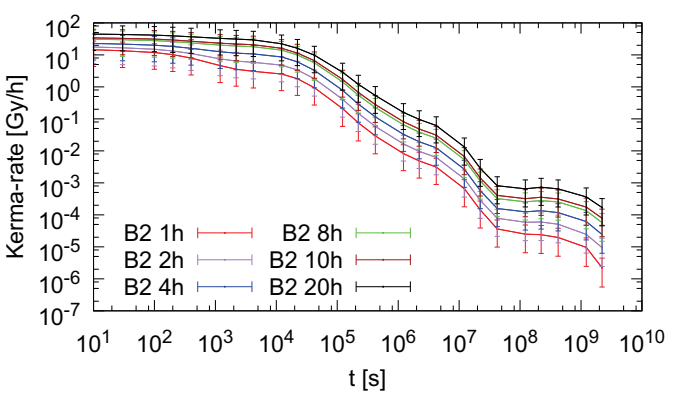

(e) B2 fuel element kerma-rate to sample.

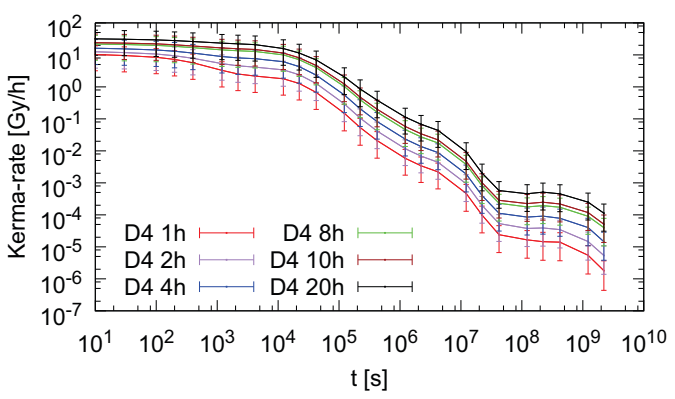

(f) D4 fuel element kerma-rate to sample.

Figure 7: Two representative fuel elements, with different activation times. X-axis denotes cooling time.

In addition to the result presented in fig. 7, individual voxel spectra were summed up and renormalized to obtain an integral $\gamma$-spectrum of the fuel element just after the end of the irradiation (fig. 8). Results in terms of isotope contribution to the contact dose are also given, as this might be important, when dealing with decomposition of neutron activated materials (fig. 9).

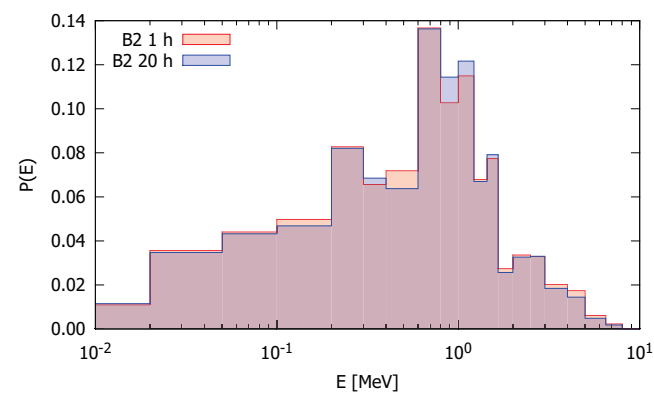

Figure 8: B2 fuel element $\gamma$-ray spectra after $1 \mathrm{~h}$ and $20 \mathrm{~h}$ of operation at full reactor power.

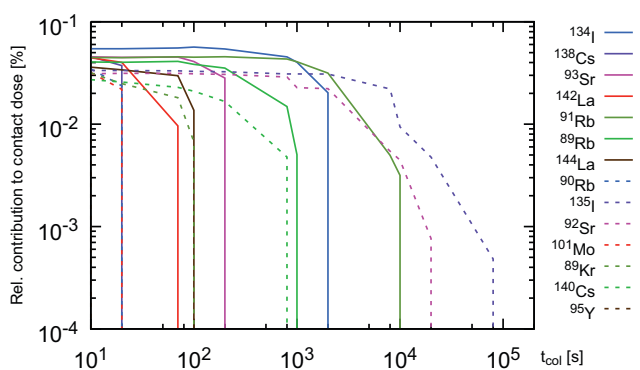

Figure 9: Isotope contributions to the contact dose in B2 element, after $20 \mathrm{~h}$ f operation at full reactor power.

\subsection{Element burn-up of $1 \mathrm{MW} \mathrm{d}$ and $10 \mathrm{MW} \mathrm{d}$}

Longer irradiation of fresh nuclear fuel in terms of burn-up of $1 \mathrm{MW} \mathrm{d}$ and $10 \mathrm{MW}$ d were also simulated, to account for accomulation of long lived isotopes, and to observe the bulk activity of the fuel elements, regardles of intraday operation. $40 \mathrm{~h} / \mathrm{w}, 8 \mathrm{~h} \mathrm{~d}^{-1}$ at full reactor power operation was simulated on each individual representative fuel element, until a sufficient burn-up was achieved. Activity and $\gamma$-ray spectrum per fuel element, as well as kerma-rate and dose-rate on the silicon irradiation sample for each fuel element were calculated (fig. 10). Per element isotopic contribution to contact dose (fig. 12) and $\gamma$ spectrum (fig. 11) were also calculated.

In fig. 10 we can observe the majority of long lived isotopic contribution, due to the fact, that the shortlived isotopes decay quickly, with all the elements having similar activities, kerma-rate and dose-rate contributions to the silicon sample after some $1 \times 10^{7} \mathrm{~s}$. However, an $\times 10$ increase it that region with all elements being approximately equal is observed, when comparing two different burn-ups, showing a linear-rate of production of long-lived isotopes, which is consistent. 


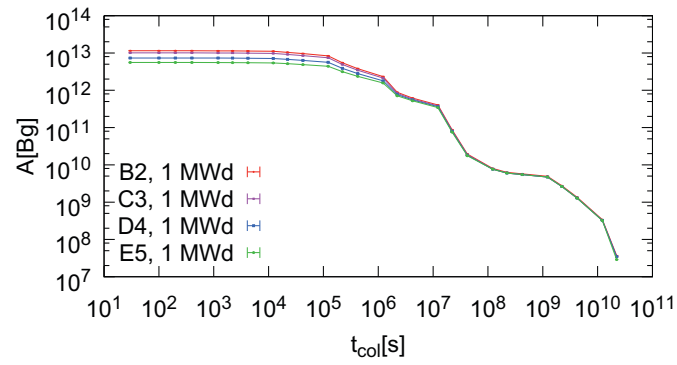

(a) Representative fuel element activity after $1 \mathrm{MWd}$ burn-up.

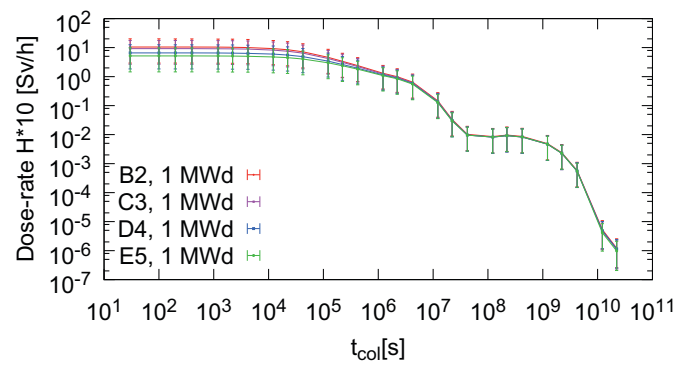

(c) $H^{*} 10$ dose-rate on silicon sample after $1 \mathrm{MW}$ d fuel element burn-up.

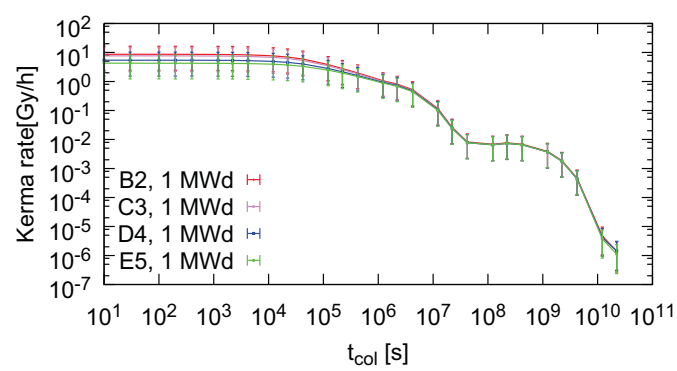

(e) Kerma-rate on silicon sample after $1 \mathrm{MW}$ d fuel element burn-up.

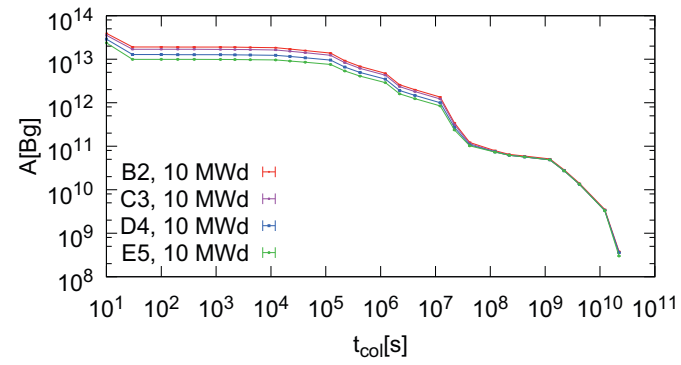

(b) Representative fuel element activity after $10 \mathrm{MW} \mathrm{d}$ burn-up.

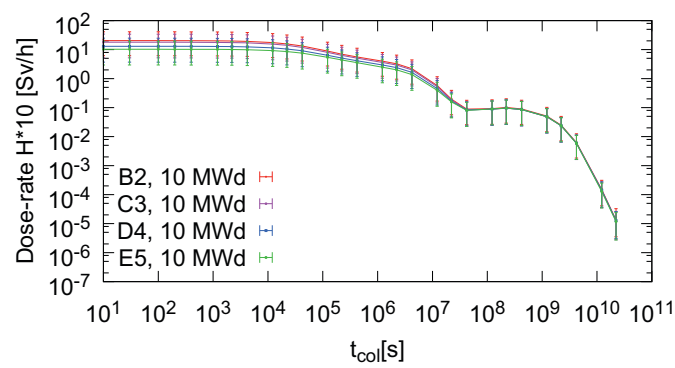

(d) $H^{*} 10$ dose-rate on silicon sample after $10 \mathrm{MW} \mathrm{d}$ fuel element burn-up.

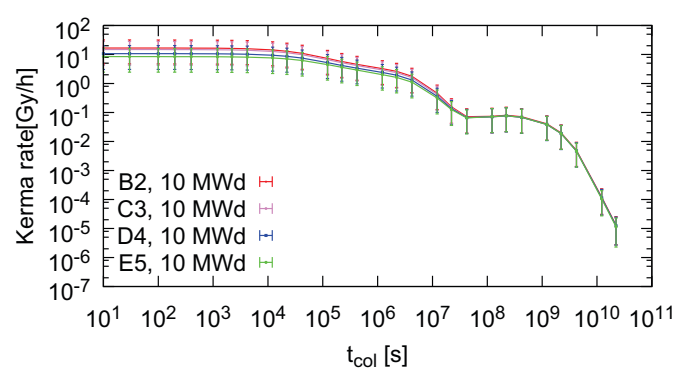

(f) Kerma-rate on silicon sample after $10 \mathrm{MW}$ d fuel element burn-up.

Figure 10: Fuel elements activity, kerma-rate and dose-rate on the silicon sample after $1 \mathrm{MW} d$ and $10 \mathrm{MW}$ d burn-up.

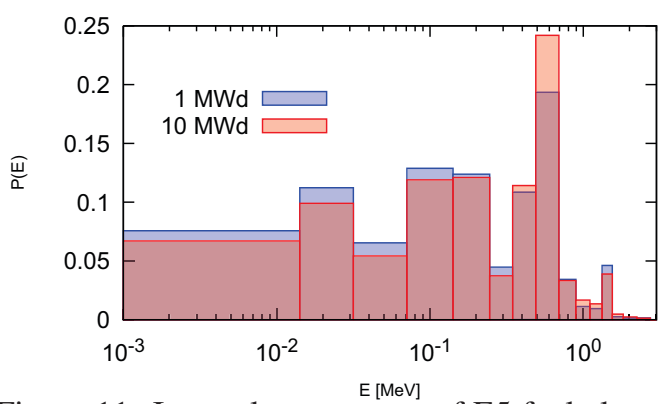

Figure 11: Integral $\gamma$ spectrum of E5 fuel element after $1 \mathrm{MW} d$ and $1 \mathrm{MW} d$ irradiation.

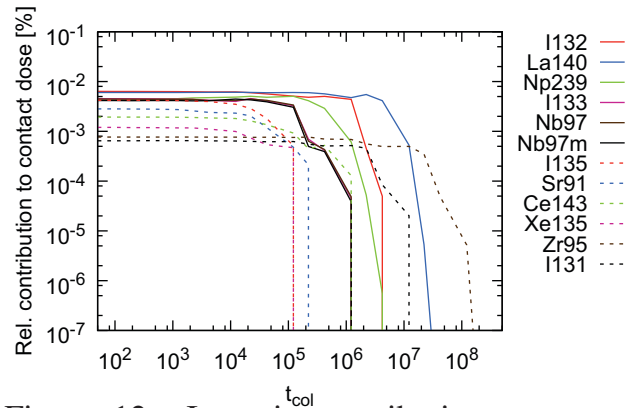

Figure 12: Isotopic $^{\mathrm{t}_{\text {col }}}$ contribution to contact dose after $1 \mathrm{MW}$ d of B2 fuel element. 


\section{Summary}

An R2S method script was developed for calculation of delayed $\gamma$-rays to improve photon characterization of irradiation facilities. JSI TRIGA nuclear fuel analysis on delayed gamma ray emission and dose deposition in was performed. Since then initial measurements on dose field around fuel element, irradiated for several hours were performed, showing agreement with dose calculations within the dose-rate distribution denoted on the graphs. To further evaluate on the code results, smaller samples around the irradiated elements should be used with precisely known positions and times after irradiation. Such measurements, utilizing different $\gamma$ dozimeters, such as TLD, OSLD, RadFET, etc.. will be performed in the next two years, to evaluate high $\gamma$ dose-rate measurement techniques inside mixed neutron and $\gamma$ fields and evaluate computational results with measurements.

The script for the R2S is still in its initial stages of the development, as several steps have not yet been automated. Also a more general script version should be made, where neutron spectra and flux voxels will be subdivided into their respective cell parts. Also model segmentation should be performed in a way, that neutron spectrum and flux do not change significantly throughout the voxel. Estimates on mesh division have thus far been made by hand, instead a computer code would be developed for full automation and accurate representation of actual state. The code will be used for $\gamma$-field characterization of the TRIGA reactor allowing its utilization for $\gamma$ radiation hardness studies. Moreover , the code could be later used to study activation of reactor components during it lifetime cycle for decomissioning purposes, to increase nuclear waste storage facilities utilization.

\section{References}

[1] L. Snoj, G. Žerovnik, A. Trkov, Applied Radiation and Isotopes 70, 483 (2012)

[2] G. Žerovnik, T. Kaiba, V. Radulovic, A. Jazbec, S. Rupnik, L. Barbot, D. Fourmentel, L. Snoj, Applied Radiation and Isotopes 96, 27 (2015)

[3] L. Snoj, B. Smodiš, 45 years of TRIGA Mark II in Slovenia, in Proceedings of the International Conference Nuclear Energy for New Europe (2011)

[4] Recent results of the ATLAS Upgrade Planar Pixel Sensors RED Project (Proceedings of science, 2011)

[5] K. Ambrožič, L. Snoj, G. Žerovnik (2016), to be published this year

[6] V. Radulović, D. Fourmentel, L. Barbot, J.F. Villard, T. Kaiba, Ž. Gašper, L. Snoj, Nuclear Instruments and Methods in Physics Research Section A: Accelerators, Spectrometers, Detectors and Associated Equipment 804, 149 (2015)

[7] D. Fourmentel, V. Radulović, L. Barbot, J.F. Villard, G. Žerovnik, L. Snoj, M. Tarchalski, K. Pytel, F. Malouch, Delayed Gamma Measurements in Different Nuclear Research Reactors Bringing Out the Importance of the Delayed Contribution in Gamma Flux Calculations, in Advancements in Nuclear Instrumentation Measurement Methods and their Applications, ANIMMA (Instituto de Plasmas e Fusão Nuclear, Lisbon, 2015)

[8] R. Villari, U. Fischer, F. Moro, P. Pereslavtsev, L. Petrizzi, S. Podda, A. Serikov, Fusion Engineering and Design 89, 2083 (2014), proceedings of the 11th International Symposium on Fusion Nuclear Technology-11 (ISFNT-11) Barcelona, Spain, 15-20 September, 2013

[9] A. Davis, Ph.D. thesis, University of Birmingham (2010)

[10] P. Sauvan, J.P. Catalán, F. Ogando, R. Juárez, J. Sanz, IEEE Transactions on Nuclear Science 63, 375 (2016)

[11] G. Rossum, Tech. rep., Amsterdam, The Netherlands, The Netherlands (1995) 
[12] T. Goorley, M. James, T. Booth, F. Brown, J. Bull, L. Cox, J. Durkee, J. Elson, M. Fensin, R. Forster et al., Nuclear Technology 180, 298 (2012)

[13] J.C. Sublet C., J.W. Eastwood, J.G. Morgan, The FISPACT-II User Manual, UK Atomic Energy Authority, Culham center for fusion energy, issue 6 edn. (2016)

[14] J. Hale, Radiology 111, 716 (1974) 